\title{
Roles of c-type cytochromes in respiration in Neisseria meningitidis
}

\begin{abstract}
Correspondence
James W. B. Moir

jm46@york.ac.uk
\end{abstract}

Received 7 May 2008

Revised 6 June 2008

Accepted 16 June 2008

\author{
Manu Deeudom, ${ }^{1,2}$ Michael Koomey ${ }^{3}$ and James W. B. Moir ${ }^{1}$ \\ ${ }^{1}$ Department of Biology (Area 10), University of York, Heslington, York Y010 5YW, UK \\ ${ }^{2}$ Department of Microbiology, Faculty of Medicine, Chiangmai University, Chiangmai 50200, \\ Thailand \\ ${ }^{3}$ Department of Molecular Biosciences, Centre for Molecular Biology and Neuroscience, University \\ of Oslo, 0316 Oslo, Norway
}

\begin{abstract}
Three c-type cytochromes were identified in Neisseria meningitidis, based on predictions from genome sequences, that were hypothesized to be involved in electron transport to terminal electron acceptor reductases for oxygen (the cytochrome $c b b_{3}$ oxidase) and nitrite (the nitrite reductase, AniA). Mutants were generated by allelic exchange with disrupted copies of the genes encoding these cytochromes and the phenotypes of the resultant mutants analysed. It was found that cytochrome $c_{5}$ is required for in vivo nitrite reductase activity, whereas cytochromes $c_{x}$ and $c_{4}$ are both required for efficient growth using oxygen as an electron acceptor. Mutants in $c_{x}, c_{4}$, and $c_{x}+c_{4}$ have a decreased capacity to reduce oxygen, but there is a background oxygenreduction activity, indicating that there may be other routes for electron transfer from the cytochrome $b c_{1}$ complex to the cytochrome $c b b_{3}$ oxidase, whereas cytochrome $c_{5}$ appears to be the sole route of electrons to the nitrite reductase in $N$. meningitidis. Interestingly, cytochrome $c_{x}$ is highly similar to a domain of copper nitrite reductases from various proteobacteria, whereas cytochrome $c_{5}$ has high identity with a domain of the cytochrome $c b b_{3}$ oxidase of Neisseria gonorrhoeae, yet these two proteins function in oxygen respiration and nitrite respiration, respectively. This highlights a limitation of predicting protein function from similarity to known proteins, i.e. very closely related protein domains in different organisms can have different redox partners.
\end{abstract}

\section{INTRODUCTION}

The $\beta$-proteobacterium Neisseria meningitidis is commonly identified as part of the commensal flora of the human nasopharyngeal mucosa. Very occasionally colonization is followed by invasion of the bloodstream by N. meningitidis, leading to life-threatening illness in the form of meningitis or septicaemia (van Deuren et al., 2000). The nasopharyngeal mucosa is habitat to a wide variety of other bacteria, including both aerobes and anaerobes (Brook, 2003). In previous work, we have determined that under aerobic conditions $N$. meningitidis is able to support respiration by reducing oxygen to water via the enzyme cytochrome $c b b_{3}$ oxidase (which is the only oxygen reductase in $N$. meningitidis), and that when oxygen becomes limiting the bacterium expresses genes encoding a nitrite reductase (aniA) and a nitric oxide reductase (nor $B)$ that collectively catalyse the respiratory reduction of nitrite to nitrous oxide (Anjum et al., 2002). N. meningitidis is able to employ this partial denitrification pathway to support growth under microaerobic conditions in the presence of nitrite (Rock et al., 2005).

We have proposed an organization for the respiratory chain of $N$. meningitidis, based on genome sequence analysis and experimental analysis with specific inhibitors (Deeudom et al., 2006). The nitric oxide reductase of $N$. meningitidis appears more similar to the quinol-oxidizing nitric oxide reductase (qNOR) than the cytochrome $c$-oxidizing NOR (cNOR) (de Vries \& Schröder, 2002), and hence it was proposed that the nitric oxide reductase receives its electrons directly from the ubiquinone pool. This was confirmed experimentally by showing that nitric oxide reduction is insensitive to the cytochrome $b c_{1}$ complex inhibitor myxothiazol. Contrastingly, the reduction of the other two electron acceptors (oxygen and nitrite) is very sensitive to myxothiazol. The enzymes responsible for these reductase reactions typically receive their electrons from $c$-type cytochromes in other micro-organisms, and hence it was proposed that these two enzymes terminate the electron-transport chain downstream of the cytochrome $b c_{1}$ complex and c-type cytochromes (Deeudom et al., 2006). The genome of $N$. meningitidis MC58 reveals the presence of genes encoding three putative $c$-type cytochromes that might mediate the transfer of electrons between the cytochrome $b c_{1}$ complex and the reductases for oxygen and nitrite. These three $c$-type cytochromes are conserved within the genomes of other $N$. meningitidis strains and in Neisseria gonorrhoeae. 
Gene NMB0717 (from N. meningitidis MC58) is predicted to encode a periplasmic mono-haem cytochrome with a molecular mass of $12.5 \mathrm{kDa}$. We call the putative product of this gene cytochrome $c_{\mathrm{x}}$. A homologue of NMB0717, cytochrome $c_{552}$ from Thermus thermophilus, has been proposed to transfer electrons between the cytochrome $b c_{1}$ complex and $b a_{3}$ oxidase in that organism (Muresanu et al., 2006). BLAST searching reveals that the predicted protein sequences with closest similarity to NMB0717, outside of the Neisseria homologues, are predicted copper-type nitrite reductases in Bdellovibrio bacteriovorus, Pseudoalteromonas haloplanktis and several Burkholderia species. In these organisms, the gene for the copper nitrite reductase (homologous to the nitrite reductase gene, aniA, of $N$. meningitidis) is fused to a cytochrome domain homologous to NMB0717. This suggests a possible role for cytochrome $c_{\mathrm{x}}$ in transfer of electrons to nitrite reductase in $N$. meningitidis.

Gene NMB1805 is predicted to encode a periplasmic dihaem cytochrome with a molecular mass of $21.5 \mathrm{kDa}$. We call the putative product of this gene cytochrome $c_{4}$, consistent with the nomenclature for the homologous gene (also known as $c y c A$ ) from $N$. gonorrhoeae (Turner et al., 2005). Cytochrome $c_{4}$ homologues are found in many other proteobacteria and a crystal structure has been found for the protein from Pseudomonas stutzeri (Kadziola \& Larsen, 1997). Both haem groups are hexacoordinated, consistent with a role in electron transfer, rather than enzyme catalysis.

Gene NMB1677 is predicted to encode a membraneassociated di-haem cytochrome with a molecular mass of $30 \mathrm{kDa}$. We call the putative product of this gene cytochrome $c_{5}$, consistent with the nomenclature for the homologous gene (also known as $c y c B$ ) from $N$. gonorrhoeae (Turner et al., 2005). The protein contains a predicted N-terminal membrane span, followed by two soluble domains containing two covalently bound haems located within the periplasm. The second haem-containing domain of cytochrome $c_{5}$ bears a striking similarity $(74 \%$ identity) to a region of the cytochrome $c b b_{3}$ oxidase subunit III (encoded by ccoP) from N. gonorrhoeae. CcoP from $N$. gonorrhoeae is predicted to contain three haem groups, whereas $\mathrm{CcoPs}$ from other organisms that have been characterized contain just two haem domains (Pitcher \& Watmough, 2004). Indeed, the predicted CcoP from $N$. meningitidis contains two haems. The similarity of cytochrome $c_{5}$ to a domain of the oxidase immediately suggests a role for cytochrome $c_{5}$ in the electron transfer from cytochrome $b c_{1}$ to the cytochrome $c b b_{3}$ oxidase.

The possession of multiple $c$-type cytochromes as part of multiply branched respiratory chains is characteristic of many bacterial species. The unambiguous assignment of function to these cytochromes is often difficult due to their large number and apparent redundancy of function. The aim of the work presented in this paper was to investigate the roles of the three putative electron-carrier proteins of
N. meningitidis in respiration in this organism, which is an important pathogen but which may also be viewed as a good model for analysis of branched respiratory metabolism due to its relatively small number of respiratory electron-acceptor reductases.

\section{METHODS}

Bacterial strains, plasmids and growth conditions. All strains of $N$. meningitidis used in this work were derived from $N$. meningitidis MC58 (McGuinness et al., 1991) (Table 1). N. meningitidis strains were routinely cultured at $37{ }^{\circ} \mathrm{C}$, in air supplemented with $5 \% \mathrm{CO}_{2}$ on Columbia agar plus $5 \%(\mathrm{v} / \mathrm{v})$ horse blood plates, or in liquid culture in Mueller-Hinton Broth (MHB) supplemented with $10 \mathrm{mM}$ $\mathrm{NaHCO}_{3}$. Aerobic culture was carried out in $5 \mathrm{ml}$ of broth in $25 \mathrm{ml}$ Sterilin McCartney bottles shaken at 200 r.p.m. Microaerobic culture was carried out using $20 \mathrm{ml}$ of broth in a $25 \mathrm{ml}$ Sterilin McCartney bottle, shaken at 90 r.p.m. and, where appropriate, supplemented with $6 \mathrm{mM} \mathrm{NaNO}_{2}$. Growth was monitored by measuring $\mathrm{OD}_{600}$ in a Jenway 6305 spectrophotometer. For whole-cell spectroscopy experiments cultures were supplemented with $5 \mathrm{mM}$ glucose in order to provide a defined electron donor that could be included as physiological reductant in cuvettes. Antibiotics were used at the following concentrations: tetracycline $20 \mu \mathrm{g} \mathrm{ml}^{-1}$, erythromycin $50 \mu \mathrm{g} \mathrm{ml}^{-1}$, kanamycin $50 \mu \mathrm{g} \mathrm{ml}^{-1}$ and spectinomycin $50 \mu \mathrm{g} \mathrm{ml}^{-1}$.

Construction of mutant strains. The gene encoding cytochrome $c_{\mathrm{x}}$ (NMB0717) and 500 bp flanking on each side was amplified with primers $\mathrm{c552F}$ and c552R (Table 1) using Pfu polymerase (Promega). The product was cloned into pCR-Blunt II TOPO (Invitrogen). The product was cleaved with BsgI, which recognizes a site located centrally within the NMB0717 gene. Subsequently, the ends of the linearized plasmid were rendered blunt with Klenow fragment and the product was ligated with the spectinomycin resistance $\left(\mathrm{Spc}^{\mathrm{R}}\right)$ gene

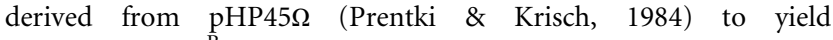
pTOPO_ $c_{\mathrm{x}}:: \mathrm{Spc}^{\mathrm{R}}$. The gene encoding cytochrome $c_{4}$ (NMB1805) and $500 \mathrm{bp}$ flanking on each side was amplified with primers c554F and c554R (Table 1) and disrupted by essentially the same method as described for cytochrome $c_{\mathrm{x}}$ except that the gene was disrupted by digestion with Bsu36I (which recognizes a site located centrally within the NMB1805 gene). Insertion of an erythromycin resistance $\left(\right.$ Ery $\left.^{\mathrm{R}}\right)$ gene [derived by PCR from a strain of $N$. meningitidis in which the gene $f n r$ was disrupted by $\operatorname{Ery}^{\mathrm{R}}$ (Rock et al., 2005)] yielded pTOPO_c4::Ery ${ }^{\mathrm{R}}$. The gene encoding cytochrome $c_{5}$ (NMB1677) and $500 \mathrm{bp}$ flanking on each side was amplified with primers c555F and c555R (Table 1) and disrupted by essentially the same method as described for cytochrome $c_{\mathrm{x}}$ except that the gene was disrupted by digestion with $X m n \mathrm{I}$ (which recognizes a site centrally within the NMB1677 gene) and insertion of a tetracycline resistance $\left(\mathrm{Tet}^{\mathrm{R}}\right)$ gene derived from Tn916, yielding pTOPO_c5:: Tet $^{\mathrm{R}}$.

The recombinant plasmids containing disrupted copies of the genes encoding cytochromes were transformed into $N$. meningitidis MC58 using the method of Bogdan et al. (2002), selecting for recombinant strains using the appropriate antibiotic selection on plates, and verifying the correct chromosomal rearrangement by PCR.

Gels and blotting. Whole-cell extracts of $N$. meningitidis were prepared by harvesting $1 \mathrm{ml}$ samples of cultures in late exponential phase by centrifugation, resuspending the pellets in $500 \mu \mathrm{l} 30 \mathrm{mM}$ Tris/HCl $(\mathrm{pH} 8)+1 \%(\mathrm{w} / \mathrm{v})$ n-dodecyl $\beta$-D-maltoside $+1 \mathrm{mg}$ lysozyme $\mathrm{ml}^{-1}+1 \mathrm{mg} \mathrm{DNase} \mathrm{Iml}^{-1}$, and subjecting the suspension to 810 cycles of freezing and thawing. Samples were separated by SDSPAGE and blotted onto nitrocellulose membranes. Membranes were stained with Ponceau $S$ to check for efficient protein transfer and 
Table 1. Strains, plasmids and oligonucleotide primers used in this work

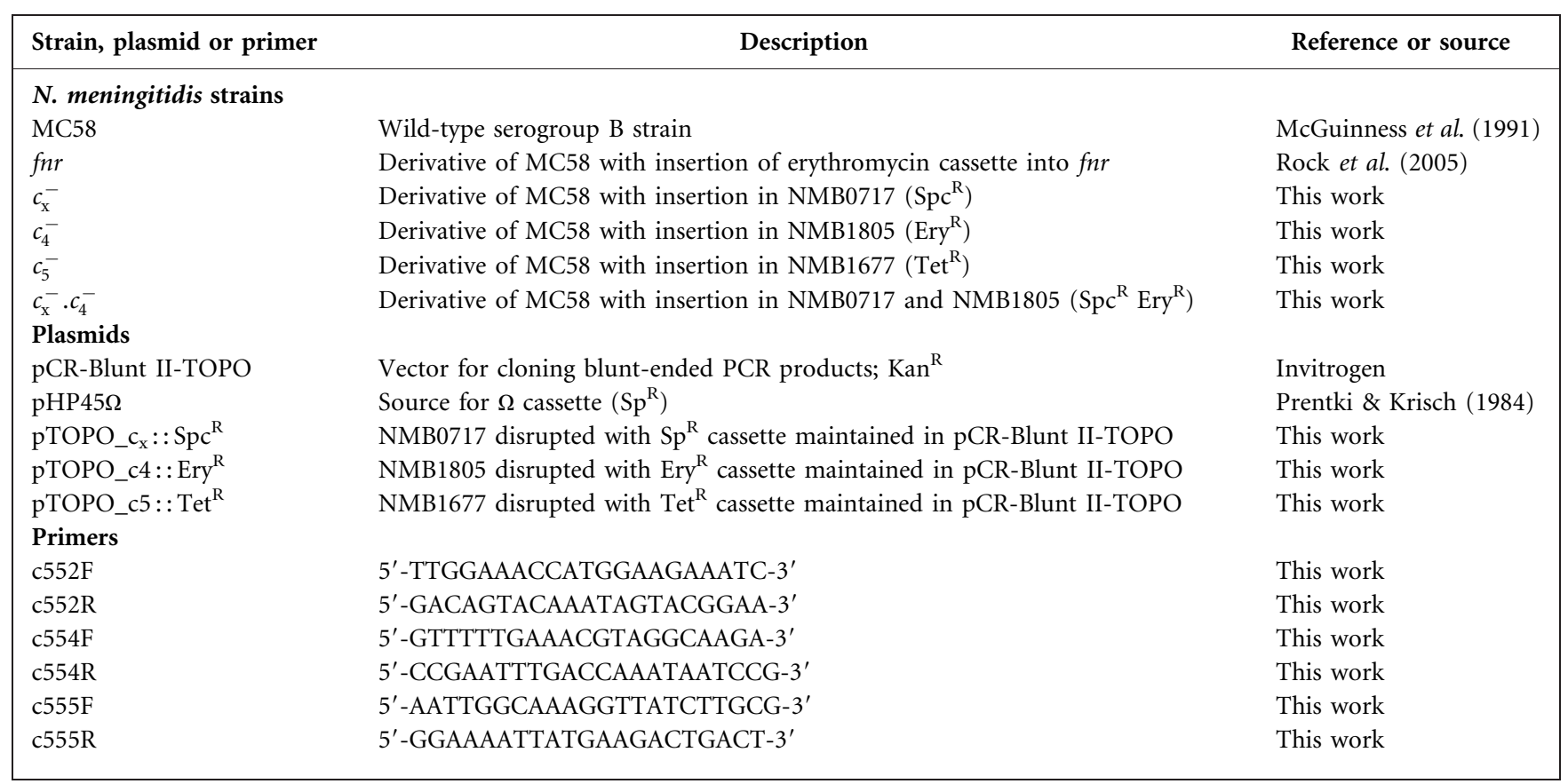

destained with water. The expression of $c$-type cytochromes was assessed using a chemiluminescence method (Vargas et al., 1993) to measure the peroxidase activity of haem groups covalently attached to protein. Five hundred microlitres of chemiluminescence detection reagents (SuperSignal West Dura substrate, Pierce) were mixed and pipetted onto the membrane, which was incubated, sandwiched between two acetate sheets, for $5 \mathrm{~min}$. The membrane was subsequently exposed to X-ray film (for $5 \mathrm{~s}$ to $5 \mathrm{~min}$ ) to achieve an optimum chemiluminescence signal. Bands due to $c$-type cytochromes were clearly visualized upon developing the film. Western blotting was used to detect expression of nitrite reductase AniA, as previously described (Rock et al., 2007).

Activity assays. Oxygen and nitric oxide were measured using electrode-based methods and nitrite was assayed colorimetrically as described previously (Rock et al., 2005). Nitrite reductase activity was assayed in whole-cell extracts using methyl viologen as electron donor as described previously (Moir et al., 1993).

Spectroscopy. The spectra of cytochromes in intact cells were measured using a Jasco V550 UV-visible spectrophotometer fitted with Integrating Reflective Sphere (ISV-469) in order to compensate for the light scattering by very high-density bacterial suspensions. Bacterial cultures grown in $\mathrm{MHB}+5 \mathrm{mM}$ glucose were harvested and resuspended in $25 \mathrm{mM}$ HEPES $+5 \mathrm{mM}$ glucose ( $\mathrm{pH} 7$ ) to an $\mathrm{OD}_{600}$ of 1.0-1.2 (in this spectrophotometer, equivalent to approx. 5-10 mg protein $\mathrm{ml}^{-1}$ ). Spectral measurements were carried out in $1 \mathrm{ml}$ cell suspensions in $1.4 \mathrm{ml}$ quartz cuvettes with sealed caps (117.100F-QS, Hellma). Each spectral measurement was taken five times to allow averaging of multiple spectra to reduce noise. Reduction of respiratory components was achieved using the physiological electron donor glucose and leaving the cell suspension to exhaust the available oxygen over the course of $10 \mathrm{~min}$. Oxygenoxidized spectra were taken following vigorous shaking of the cell suspension. Oxidation of the suspension by nitrite was achieved by addition of $5 \mathrm{mM}$ nitrite (in a total volume of $5 \mu$ lo minimize dilution effect).

\section{RESULTS}

\section{Construction and confirmation of mutants in cytochromes $c_{x}, c_{4}$ and $c_{5}$}

Mutant strains deficient in $c_{\mathrm{x}}, c_{4}, c_{5}$ and $c_{\mathrm{x}}+c_{4}$ were generated following transformation of the constructs described above into N. meningitidis MC58. In each case, the replacement of the wild-type copy of the gene with the copy containing an antibiotic resistance cassette was confirmed by PCR (data not shown). In order to obtain further biochemical confirmation, whole-cell extracts generated from each of the strains were run on SDSPAGE, blotted onto nitrocellulose and stained for haem (Fig. 1a). The lane containing extract from strain $N$. meningitidis $c_{5}^{-}$reveals the absence of a cytochrome band with molecular mass of approximately $33 \mathrm{kDa}$, consistent with the absence of cytochrome $c_{5}$ (which has a predicted molecular mass of $30 \mathrm{kDa}$ ). The lanes containing extracts from strains $N$. meningitidis $c_{4}^{-}$and $c_{\mathrm{x}}^{-} \cdot c_{4}^{-}$lacked an intense band with a molecular mass of approximately $23 \mathrm{kDa}$, consistent with the absence of cytochrome $c_{4}$ (predicted molecular mass $21.5 \mathrm{kDa}$ ) from these strains. There is no difference in the appearance of lanes between $c_{4}^{-}$and $c_{\mathrm{x}}^{-} \cdot c_{4}^{-}$or between MC58 and $c_{\mathrm{x}}^{-}$, indicating that a band due to cytochrome $c_{\mathrm{x}}$ cannot be observed by this method.

UV-visible reduced-minus-oxidized spectroscopy of intact cells of N. meningitidis wild-type yielded spectra consistent with the presence of $c$-type ( $\alpha$ band located near $550 \mathrm{~nm}$ ) and $b$-type ( $\alpha$ band shoulder near $560 \mathrm{~nm}$ ) cytochromes in this organism (Fig. 2). Comparison of wild-type versus strains deficient in $c_{\mathrm{x}}, c_{4}$ and $c_{5}$ indicates the absence of 
(a)

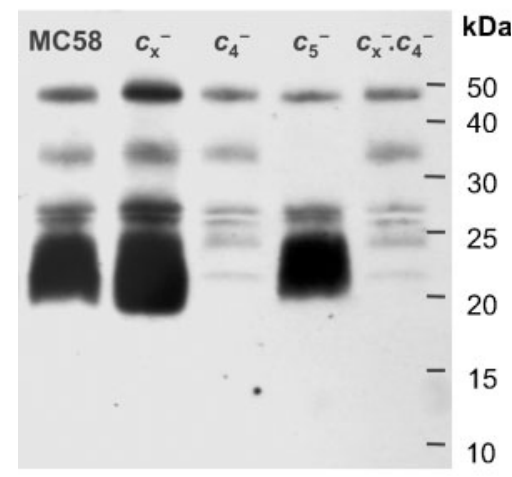

(b)

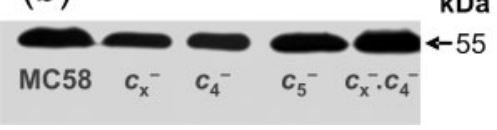

Fig. 1. (a) Haem-stained blot of $N$. meningitidis extracts shows altered expression of cytochromes. Samples $(13 \mu \mathrm{g})$ of total cell extracts from $N$. meningitidis wild-type MC58 and the mutants indicated (aerobically grown) were separated by $15 \%$ SDSPAGE, blotted and stained for haem. (b) Western blot with antiAniA antibodies. The $N$. meningitidis strains were grown under microaerobic conditions plus nitrite. Gels were run as for (a).

specific cytochromes in each of the constructs (Fig. 2). The overall intensities of the redox difference spectra were decreased for the cytochrome-deficient strains compared to the wild-type. Furthermore, the positions of the $\alpha$ bands in these difference spectra are shifted compared to the wildtype. For N. meningitidis MC58, the $\alpha$ band is positioned at $553 \mathrm{~nm}$, whilst the $c_{\mathrm{x}}^{-}$and $c_{5}^{-}$mutants have their $\alpha$ peak at $552 \mathrm{~nm}$ and the $c_{4}^{-}$mutant has an $\alpha$ band at $555 \mathrm{~nm}$. The $\alpha$

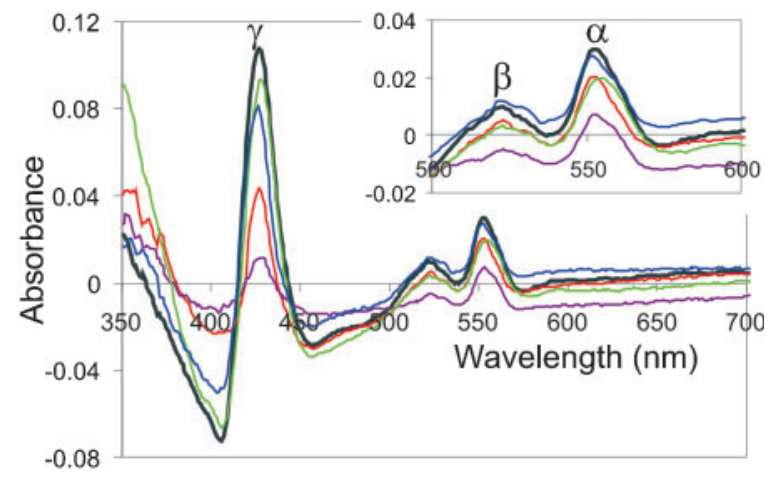

Fig. 2. UV-visible redox spectra of intact $N$. meningitidis. Spectra are endogenously reduced minus oxygen-oxidized spectra of $N$. meningitidis wild-type MC58 (black) and mutants $c_{x}^{-}$(red), $c_{4}^{-}$ (green), $c_{5}^{-}$(blue), and $c_{x}^{-} \cdot c_{4}^{-}$(purple). The positions of spectral peaks $\alpha, \beta$ and $\gamma$ are marked. For each cell suspension, the $\mathrm{OD}_{600}$ for the endogenously reduced sample was 1.0. band of the $c_{\mathrm{x}}^{-} \cdot c_{4}^{-}$mutant is centred at $553 \mathrm{~nm}$. It is notable that the mutation in $c_{\mathrm{x}}$ causes a shift in the $\alpha$ band as well as a decrease in spectral intensity (for MC58 versus $c_{\mathrm{x}}^{-}$and for $c_{4}^{-}$versus $c_{\mathrm{x}}^{-} \cdot c_{4}^{-}$), providing evidence that cytochrome $c_{\mathrm{x}}$ is expressed in wild-type $N$. meningitidis and absent from the $c_{\mathrm{x}}$ mutant strains (despite giving no band on haem-stain blots).

\section{Growth properties of cytochrome-deficient mutants of $\boldsymbol{N}$. meningitidis}

$N$. meningitidis MC58, $c_{\mathrm{x}}^{-}, c_{4}^{-}, c_{5}^{-}$and $c_{\mathrm{x}}^{-} . c_{4}^{-}$were cultured under aerobic conditions and under microaerobic conditions in the presence and absence of $5 \mathrm{mM}$ nitrite (Fig. 3). The growth rate of the $c_{5}^{-}$mutant was virtually the same as that of the wild-type during the exponential phase in aerobic culture (doubling time of $43 \mathrm{~min}$ ), whereas the $c_{\mathrm{x}}^{-}, c_{4}^{-}$and $c_{\mathrm{x}}^{-} \cdot c_{4}^{-}$mutants grew relatively poorly, with doubling times of $63 \mathrm{~min}, 130 \mathrm{~min}$ and $174 \mathrm{~min}$, respectively (Fig. 3a). N. meningitidis MC58, $c_{\mathrm{x}}^{-}, c_{4}^{-}$and $c_{5}^{-}$grew at similar rates to one another under microaerobic conditions in the absence but not the presence of nitrite (Fig. 3b, c). In the presence of nitrite, N. meningitidis MC58, $c_{\mathrm{x}}^{-}, c_{4}^{-}$and $c_{\mathrm{x}}^{-} \cdot c_{4}^{-}$had an exponential phase of growth, as expected for cultures that have nutrient sufficiency when nitrite is present, whereas the $c_{5}^{-}$mutant had a linear growth curve, as would be expected for a strain that is limited by availability of a nutrient that is supplied at a constant rate, such as oxygen (Fig. 3c). This has been observed previously for strains of $N$. meningitidis that are unable to denitrify (Rock et al., 2005), and suggests that under these growth conditions the cytochrome $c_{5}$-deficient strain grows using the limiting nutrient oxygen and is unable to denitrify. Measurements of nitrite remaining in culture medium corroborated this proposition, since nitrite disappeared during the course of growth of all strains except $N$. meningitidis $c_{5}^{-}$(Fig. 3d). It is notable that $N$. meningitidis $c_{\mathrm{x}}^{-}, c_{4}^{-}$and $c_{\mathrm{x}}^{-} \cdot c_{4}^{-}$grew to lower final cell densities than did N. meningitidis MC58, and yet utilized nitrite as quickly as or quicker than the wild-type. This is consistent with the relatively poor ability of these strains to grow aerobically (Fig. 3a), and indicates that under denitrifying conditions these strains use nitrite as the major electron sink for respiration whereas the wild-type combines nitrite and oxygen utilization simultaneously.

To test whether the impaired aerobic growth observed in mutants deficient in $c_{\mathrm{x}}, c_{4}$ and $c_{\mathrm{x}}+c_{4}$ was due to an inability to metabolize oxygen, the rate of oxygen uptake was measured in suspensions of $N$. meningitidis strains using a Clark-type oxygen electrode. The rate of oxygen uptake was measured for $c_{\mathrm{x}}^{-}$and $c_{4}^{-}$strains relative to wild-type following aerobic growth. The rates of oxygen uptake were $83 \pm 5 \%$ and $59 \pm 5 \%$ of the wild-type rate in strains $c_{\mathrm{x}}^{-}$and $c_{4}^{-}$respectively. The growth of $N$. meningitidis $c_{\mathrm{x}}^{-} \cdot c_{4}^{-}$was so poor aerobically that we cultured the wild-type and $c_{\mathrm{x}}^{-} \cdot c_{4}^{-}$strains under denitrifying conditions to analyse the relative oxygen respiration 

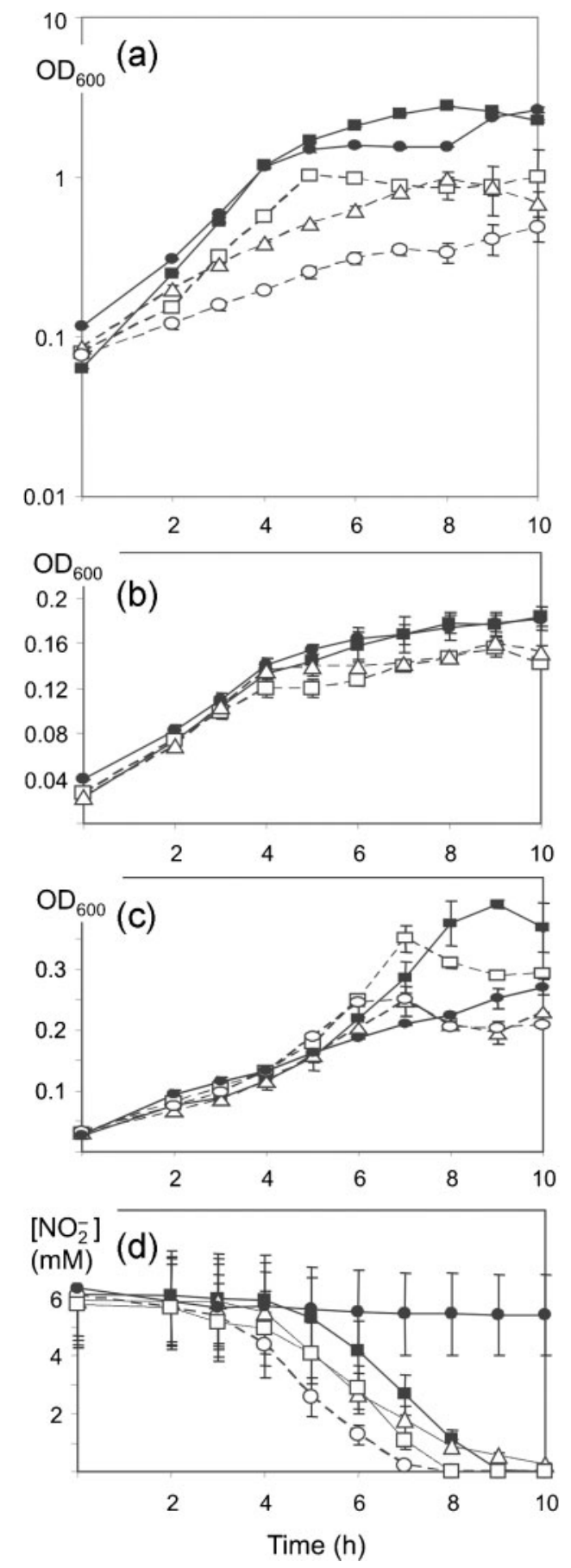

Fig. 3. Effect of deficiency in cytochromes on growth of $N$. meningitidis. Cultures of $N$. meningitidis wild-type MC58 ( $\mathbf{\square}-\mathbf{\square})$ and mutants $c_{\mathrm{x}}^{-}(\square---\square), c_{4}^{-}(\triangle---\triangle), c_{5}^{-}(\mathbf{\bullet}-\bullet)$ and $c_{\mathrm{x}}^{-} \cdot c_{4}^{-}$ $(\bigcirc--\bigcirc)$ were grown under aerobic conditions (a), under microaerobic conditions without nitrite (b) and under microaerobic conditions plus $6 \mathrm{mM}$ nitrite (c). In each case growth was monitored by measuring $\mathrm{OD}_{600}$. Nitrite disappearance was monitored for microaerobic cultures supplemented with nitrite (d). rate in this strain. The rate of oxygen uptake was $45 \pm 5 \%$ of the wild-type rate in the $c_{\mathrm{x}}^{-} \cdot c_{4}^{-}$strain. Clearly both cytochromes $c_{\mathrm{x}}$ and $c_{4}$ are involved in enabling $N$. meningitidis to utilize oxygen and to grow aerobically.

$N$. meningitidis $c_{5}^{-}$failed to utilize nitrite during incubation under microaerobic conditions with nitrite. To determine whether this was due to an inability to respire nitrite or nitric oxide we grew strains MC58 and $c_{5}^{-}$ microaerobically in the absence of nitrite and then followed the accumulation of nitric oxide using an $\mathrm{NO}$ electrode after the addition of nitrite (Fig. 4). No nitric oxide accumulated from the $c_{5}^{-}$strain, whereas, as expected, it accumulated immediately on adding nitrite to the wildtype. This indicates that the lesion in the $c_{5}$ mutant relates to nitrite reduction, not nitric oxide reduction. To assess whether the defect in nitrite reduction in $N$. meningitidis $c_{5}^{-}$might be due to low nitrite reductase expression and/or activity rather than to a break in the electron-transport chain to the nitrite reductase we measured nitrite reductase expression and activity. Expression of nitrite reductase was assessed by Western blotting (Fig. 1b), which showed that AniA is expressed at similar levels in all strains. Using a methyl viologen-linked nitrite reductase assay in total cell extracts we found that N. meningitidis MC58 and $c_{5}^{-}$had similar overall rates of nitrite reductase activity (data not shown). In the absence of cytochrome $c_{5}$ the electrontransport chain to nitrite reductase is disabled, presumably because cytochrome $c_{5}$ is an electron carrier between the cytochrome $b c_{1}$ complex and AniA nitrite reductase.

\section{Spectroscopic analysis of oxidation of $\boldsymbol{N}$. meningitidis cytochromes by oxygen versus nitrite}

Like oxygen, nitrite was found to be capable of oxidizing cytochromes in N. meningitidis MC58 (but only after

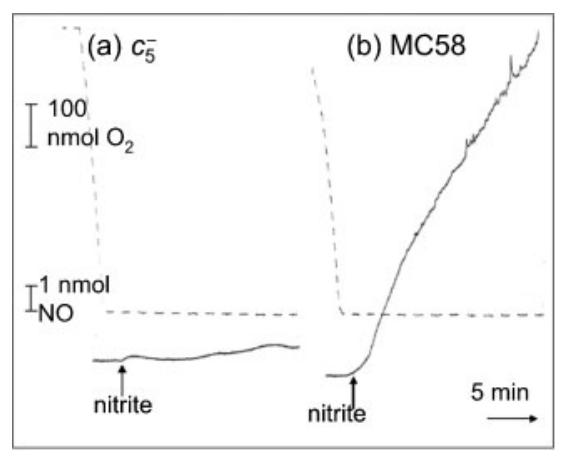

Fig. 4. Nitric oxide production in N. meningitidis wild-type MC58 but not the $c_{5}^{-}$mutant strain. N. meningitidis MC58 and $c_{5}^{-}$were grown under microaerobic conditions, and a $5 \mathrm{ml}$ cell suspension was allowed to become anaerobic in a water-jacketed electrode chamber at $37{ }^{\circ} \mathrm{C}$. Oxygen was monitored with a Clark-type electrode (dashed line). Nitric oxide was monitored with an NO electrode (solid line) before and after the addition of $5 \mathrm{mM}$ nitrite. 
growth under microaerobic conditions plus nitrite) (Fig. 5). The redox difference spectrum following oxidation by nitrite is less intense than that following oxidation by oxygen, consistent with nitrite being a less good oxidant than oxygen. Furthermore, the positions of the spectral maxima are different (the $\alpha$ band is located at $560 \mathrm{~nm}$ ), indicating that oxidation by nitrite brings about predominantly the oxidation of $b$-type cytochromes. The redox difference spectra following oxidation by nitric oxide (provided by treating cells with $1 \mathrm{mM}$ DEA-NONOate) gives similar spectra to those seen on oxidation by nitrite. Redox difference spectra of $N$. meningitidis $c_{5}^{-}$following treatment with nitrite showed no features, consistent with this strain possessing no significant electron-transport chain to nitrite reductase (Fig. 5).

\section{DISCUSSION}

In this study we investigated the roles of the three putative electron-carrier $c$-type cytochromes of $N$. meningitidis in respiratory electron transport during oxygen respiration and denitrification of nitrite to nitrous oxide. The evidence establishes that cytochrome $c_{\mathrm{x}}$ and cytochrome $c_{4}$ are important for oxygen reduction and that cytochrome $c_{5}$ is necessary for the reduction of nitrite to nitric oxide. These findings run counter to the expectations based on similarity of the cytochromes to other proteins in sequence databases. Most notably, cytochrome $c_{\mathrm{x}}$ is highly homologous to a cytochrome-containing domain of copper nitrite reductases from various proteobacteria, which had indicated that cytochrome $c_{\mathrm{x}}$ might be involved in electron transfer to nitrite reductase in $N$. meningitidis, whereas the experimental finding was that mutation of cytochrome $c_{\mathrm{x}}$ has no deleterious effect on nitrite reduction. Similarly,

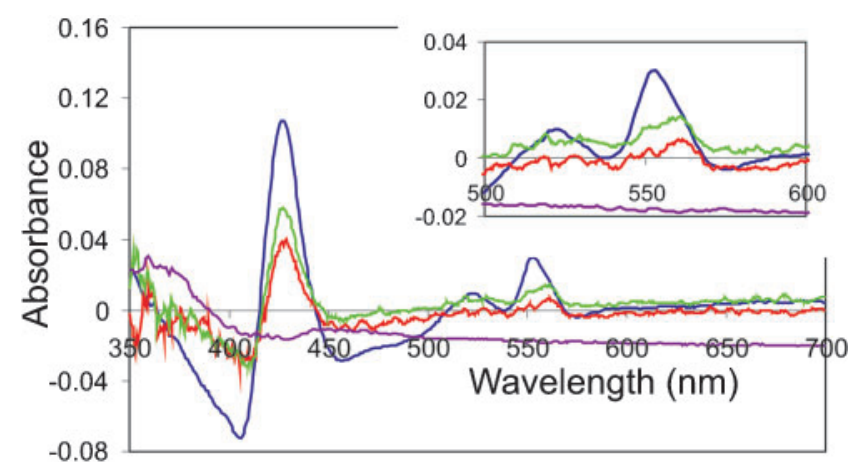

Fig. 5. UV-visible redox spectra of intact $N$. meningitidis wildtype MC58 and mutant $C_{5}^{-}$. Spectra are endogenously reduced minus oxygen-oxidized spectra of strain MC58 (blue), reduced minus nitrite-oxidized spectra of strain MC58 (red), reduced minus nitric oxide-oxidized spectra of strain MC58 (green), and reduced minus nitrite-oxidized spectra of strain $c_{5}^{-}$(purple). For each cell suspension, the $\mathrm{OD}_{600}$ for the endogenously reduced sample was equal to 1.0 . cytochrome $c_{5}$ (identified experimentally as required for nitrite reduction) is very similar to a domain of the cytochrome $c b b_{3}$ oxidase of $N$. gonorrhoeae, which had suggested that this protein might be important for oxygen respiration. This is an important caveat to bear in mind when predicting function of electron-transport proteins, i.e. very closely related protein domains in different organisms can have different redox partners.

The simplest explanation of the data we have obtained is that cytochrome $c_{5}$ is a mediator that carries electrons from the cytochrome $b c_{1}$ complex to the AniA nitrite reductase. Cytochromes $c_{\mathrm{x}}$ and $c_{4}$ are not required for the reduction or oxidation of $c_{5}$. Is it feasible that cytochrome $c_{5}$ might be a direct electron donor to AniA nitrite reductase? AniA consists of a trimer of water-soluble subunits (Boulanger \& Murphy, 2002) associated with the outer membrane via covalent attachment of $\mathrm{N}$-terminal cysteine residues to fatty acid moieties (Clark et al., 1987; Hoehn \& Clark, 1992). It is presumed to be associated with the inner leaflet of the outer membrane, and hence located within the periplasmic compartment. To reduce AniA and drive nitrite reduction, electrons must be transported across the periplasm from the cytochrome $b c_{1}$ complex (in the inner membrane) to AniA. Can cytochrome $c_{5}$ span this gap? Analysis of the sequence of cytochrome $c_{5}$ indicates that it arose by a duplication event from an ancestral gene encoding a monohaem protein. There is no structure available for a dihaem cytochrome $c_{5}$, but structural information is available for a homologous monohaem cytochrome $c_{5}$ from Shewanella putrefaciens (Bartalesi et al., 2002). This globular protein has a diameter of approximately $30 \AA(3 \mathrm{~nm})$, indicating that the dimeric cytochrome $c_{5}$ from $N$. meningitidis may span approximately $60 \AA$. N. meningitidis cytochrome $c_{5}$ is predicted to be attached to the membrane via a transmembrane helix, and thus it is anticipated that it can form a structure which protrudes up to approximately $60 \AA$ into the periplasm from the inner membrane. The ingress of electrons to AniA occurs via a blue copper centre which is accessed from a site on the surface of AniA which is approximately $45 \AA$ from the $\mathrm{N}$-terminal face of the soluble protein structure (Boulanger \& Murphy, 2002). Thus a complex of the globular domains of cytochrome $c_{5}$ and AniA could span some $105 \AA$ across the periplasm. The width of the Gramnegative periplasm is approximately $170 \AA$, based on electron microscopy measurements (Matias et al., 2003) and calculations based on the interaction of innermembrane protein AcrB with outer-membrane protein TolC (Tamura et al., 2005). Towards the $\mathrm{N}$ terminus of the mature AniA polypeptide there is a 35-40 residue region rich in alanine, proline and glutamate that may form an elongated unstructured linker that will allow AniA to get access to electrons from cytochrome $\mathcal{c}_{5}$. It will be of interest to shorten this 'linker region' to determine whether it is required for allowing AniA to obtain electrons.

Haem staining was used successfully to identify cytochromes $c_{4}$ and $c_{5}$ in total extracts of $N$. meningitidis 
strains, but cytochrome $c_{\mathrm{x}}$ could not be seen by this method. Haem staining relies on the peroxidase activity of the haem group, an activity that is affected by the naturation state of the protein folding around the haem group (Diederix et al., 2002). This activity varies between cytochromes and presumably is very low for cytochrome $c_{\mathrm{x}}$. Spectroscopic measurements, however, showed a significant loss of $c$-type cytochrome from the $c_{\mathrm{x}}$ mutant (Fig. 2), confirming that this is a major cytochrome in $N$. meningitidis. The additive effects of mutations in $c_{\mathrm{x}}$ and $c_{4}$ on oxygen respiration indicate that these two proteins operate as independent parallel pathways of electrons from the cytochrome $b c_{1}$ complex to the cytochrome $c b b_{3}$ oxidase (Fig. 6). Additionally, there is residual oxidase activity in a $c_{\mathrm{x}}^{-} \cdot c_{4}^{-}$mutant, indicating that there are alternative pathways to cytochrome $c b b_{3}$. Cytochrome $c_{5}$ may act as an alternative electron carrier to the oxidase, and it is noticeable that growth of a $c_{5}^{-}$mutant aerobically is slower than that of the wild-type at high optical densities in late exponential phase (Fig. 4a). We were unable to construct a $c_{\mathrm{x}}^{-} \cdot c_{4}^{-} \cdot c_{5}^{-}$triple mutant, lending support to this proposition. An alternative route might involve the outer-membrane-associated cupredoxin Laz (lipid-modified azurin) (Gotschlich \& Seiff, 1987; Kawula et al., 1987). Mutants deficient in the gene encoding Laz grew well aerobically (Wu et al., 2005; our unpublished data) but we were unable to generate a $c_{\mathrm{x}}^{-} \cdot c_{4}^{-}$.laz triple mutant (data not shown). The proposed electron-transport chain of $N$. meningitidis is summarized in Fig. 6.

Spectroscopic analysis following oxidation of reduced suspensions of intact $N$. meningitidis with oxygen, nitrite and nitric oxide indicates that on oxidation of cells with nitrite the main chromophores that are oxidized are $b$-type cytochromes, which are also oxidized directly by nitric oxide [nitric oxide reductase itself is a $b$-type cytochrome (de Vries \& Schröder, 2002)]. Whilst oxygen strongly oxidizes the cytochrome $c$ pool in steady-state cell suspensions, nitrite does not. At steady state in the presence of nitrite, the cytochrome $c$ pool remains largely reduced. Presumably this is related to the fact that nitrite is a poor oxidant relative to oxygen $\left(E_{\mathrm{NO}_{2}^{-} / \mathrm{NO}}^{\mathrm{N}^{\prime}}=0.375 \mathrm{~V}\right.$;

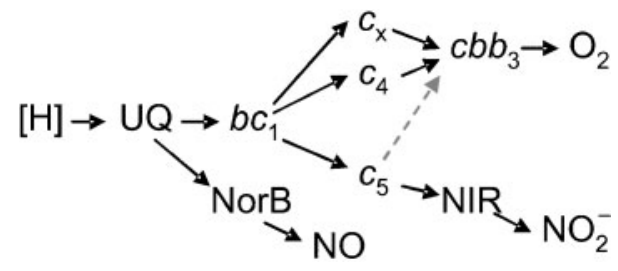

Fig. 6. Proposed electron-transport chain of $N$. meningitidis. Electron flow from cytoplasmic reductants $(\mathrm{NADH}$, succinate, etc.: shown as $[\mathrm{H}])$ to terminal electron acceptors $\mathrm{O}_{2}, \mathrm{NO}_{2}^{-}$and $\mathrm{NO}$ is shown by solid arrows. A possible alternative electron transport pathway from cytochrome $C_{5}$ to the oxidase is shown by the dashed arrow. UQ, ubiquinone; NIR, nitrite reductase.
$\left.E_{\mathrm{O}_{2} / \mathrm{H}_{2} \mathrm{O}}^{\mathrm{o}^{\prime}}=0.8 \mathrm{~V}\right)$. It may also be related to the fact that nitrite reductase draws electrons from only cytochrome $c_{5}$ whereas oxygen oxidizes cytochromes $c b b_{3}, c_{\mathrm{x}}, c_{4}$ and possibly $c_{5}$. Furthermore, this spectroscopic observation is consistent with the finding that oxygen almost completely inhibits nitrite reduction in intact cells, whereas the reverse is not the case (Rock et al., 2005); i.e. oxygen is able to outcompete nitrite for electrons at the level of cytochrome $c$.

In summary, we have identified the roles of three $c$-type cytochromes in the respiratory chain of $N$. meningitidis. Respiration is crucial for survival of $N$. meningitidis, and these cytochromes are highly conserved and extracytoplasmic, making them potential targets for the development of novel therapeutics against pathogenic neisseriae.

\section{ACKNOWLEDGEMENTS}

M.D. would like to thank the Royal Thai Government for the financial support that allowed him to study for a $\mathrm{PhD}$ under J.W.B.M. The authors are grateful to Jeff Cole, Clive Butler and Gavin Thomas for helpful discussions during the course of this work.

\section{REFERENCES}

Anjum, M. F., Stevanin, T. M., Read, R. C. \& Moir, J. W. B. (2002). Nitric oxide metabolism in Neisseria meningitidis. J Bacteriol 184, 2987-2993.

Bartalesi, I., Bertini, I., Hajieva, P., Rosato, A. \& Vasos, P. R. (2002). Solution structure of a monoheme ferrocytochrome $c$ from Shewanella putrefaciens and structural analysis of sequence-similar proteins: functional implications. Biochemistry 41, 5112-5119.

Bogdan, J. A., Minetti, C. A. \& Blake, M. S. (2002). A one step method for genetic transformation of non-piliated Neisseria meningitidis. $J$ Microbiol Methods 49, 97-101.

Boulanger, M. J. \& Murphy, M. E. (2002). Crystal structure of the soluble domain of the major anaerobically induced outer membrane protein (AniA) from pathogenic Neisseria: a new class of coppercontaining nitrite reductases. J Mol Biol 315, 1111-1127.

Brook, I. (2003). Microbial dynamics of purulent nasopharyngitis in children. Int J Pediatr Otorhinolaryngol 67, 1047-1053.

Clark, V. L., Campbell, L. A., Palermo, D. A., Evans, T. M. \& Klimpel, K. W. (1987). Induction and repression of outer membrane proteins by anaerobic growth of Neisseria gonorrhoeae. Infect Immun 55, 1359-1364.

Deeudom, M., Rock, J. \& Moir, J. W. B. (2006). Organization of the respiratory chain of Neisseria meningitidis. Biochem Soc Trans 34, 139-142.

de Vries, S. \& Schröder, I. (2002). Comparison between the nitric oxide reductase family and its aerobic relatives, the cytochrome oxidases. Biochem Soc Trans 30, 662-667.

Diederix, R. E. M., Ubbink, M. \& Canters, G. W. (2002). Peroxidase activity as a tool for studying the folding of $c$-type cytochromes. Biochemistry 41, 13067-13077.

Gotschlich, E. C. \& Seiff, M. E. (1987). Identification and gene structure of an azurin-like protein with a lipoprotein signal peptide in Neisseria gonorrhoeae. FEMS Microbiol Lett 43, 253-255.

Hoehn, G. T. \& Clark, V. L. (1992). The major anaerobically induced outer membrane protein of Neisseria gonorrhoeae, Pan1, is a lipoprotein. Infect Immun 60, 4704-4708. 
Kadziola, A. \& Larsen, S. (1997). Crystal structure of the di-haem cytochrome $c_{4}$ from Pseudomonas stutzeri determined at $2.2 \AA$ resolution. Structure 5, 203-216.

Kawula, T. H., Spinol, S. M., Klapper, D. G. \& Cannon, J. G. (1987). Localization of a conserved epitope and an azurin-like domain in the H.8 protein of pathogenic Neisseria. Mol Microbiol 1, 179-185.

Matias, V. R., Al-Amoudi, A., Dubochet, J. \& Beveridge, T. J. (2003). Cryo-transmission electron microscopy of frozen-hydrated sections of Escherichia coli and Pseudomonas aeruginosa. J Bacteriol 185, 6112-6118.

McGuinness, B. T., Clarke, I. N., Lambden, P. R., Barlow, A. K., Poolman, J. T., Jones, D. M. \& Heckels, J. E. (1991). Point mutation in meningococcal porA gene associated with increased endemic disease. Lancet 337, 514-517.

Moir, J. W. B., Baratta, D., Richardson, D. J. \& Ferguson, S. J. (1993). The purification of a $c d_{1}$-type nitrite reductase and the absence of a copper-type nitrite reductase from the aerobic denitrifier Thiosphaera pantotropha; the role of pseudoazurin as an electron donor. Eur $J$ Biochem 212, 377-385.

Muresanu, L., Pristovsek, P., Löhr, F., Maneg, O., Mukrasch, M. D., Rüterjans, H., Ludwig, B. \& Lücke, C. (2006). The electron transfer complex between cytochrome $c_{552}$ and the $\mathrm{Cu}_{\mathrm{A}}$ domain of the Thermus thermophilus $b a_{3}$ oxidase. A combined NMR and computational approach. J Biol Chem 281, 14503-14513.

Pitcher, R. S. \& Watmough, N. J. (2004). The bacterial cytochrome $\mathrm{cbb}_{3}$ oxidases. Biochim Biophys Acta 1655, 388-399.

Prentki, P. \& Krisch, H. M. (1984). In vitro insertional mutagenesis with a selectable DNA fragment. Gene 29, 303-313.
Rock, J. D., Mahnane, M. R., Anjum, M. F., Shaw, J. G., Read, R. C. \& Moir, J. W. B. (2005). The pathogen Neisseria meningitidis requires oxygen, but supplements growth by denitrification. Nitrite, nitric oxide and oxygen control respiratory flux at genetic and metabolic levels. Mol Microbiol 58, 800-809.

Rock, J. D., Thomson, M. J., Read, R. C. \& Moir, J. W. B. (2007). Regulation of denitrification genes in Neisseria meningitidis by nitric oxide and the repressor NsrR. J Bacteriol 189, 1138-1144.

Tamura, N., Murakami, S., Oyama, Y., Ishiguro, M. \& Yamaguchi, A. (2005). Direct interaction of multidrug efflux transporter AcrB and outer membrane channel TolC detected via site-directed disulfide cross-linking. Biochemistry 44, 11115-11121.

Turner, S. M., Moir, J. W. B., Griffiths, L., Smith, H. \& Cole, J. A. (2005). Genomic analysis of the c-type cytochromes of Neisseria gonorrhoeae: mutational and biochemical analysis of cytochrome $c^{\prime}$, a nitric oxidebinding lipoprotein important for adaptation to microaerobic growth and its implications for pathogenicity. Biochem J 388, 545-553.

van Deuren, M., Brandtzaeg, P. \& van der Meer, J. W. M. (2000). Update on meningococcal disease with emphasis on pathogenesis and clinical management. Clin Microbiol Rev 13, 144-166.

Vargas, C., McEwan, A. G. \& Downie, J. A. (1993). Detection of $c$-type cytochromes using enhanced chemiluminescence. Anal Biochem 209, 323-326.

Wu, H. J., Seib, K. L., Edwards, J. L., Apicella, M. A., McEwan, A. G. \& Jennings, M. P. (2005). Azurin of pathogenic Neisseria spp. is involved in defense against hydrogen peroxide and survival within cervical epithelial cells. Infect Immun 73, 8444-8448.

Edited by: R. J. M. van Spanning 